\title{
Ações de Enfermagem que contribuem para o preparo da colonoscopia: revisão integrativa
}

\section{Nursing actions aimed at preparing for colonoscopy: an integrative review}

\author{
Thaís Vasconselos Amorim' • Lara Alves Gomes ${ }^{2} \bullet$ Letícia da Silva Coelho $^{3} \bullet$ Andyara do Carmo Pinto Coelho Paiva ${ }^{4}$ \\ Anna Maria de Oliveira Salimena ${ }^{5}$ Bruna Lourenço Cassimiro $^{6} \bullet$ Suellen Fernanda de Souza Viana ${ }^{7}$ \\ Amanda Tamires Drumond Vilas Boas Tavares ${ }^{8} \bullet$ Layla Guimarães Paixão Oliveira 9 \\ Rômulo Cândido Nogueira do Nascimento ${ }^{10}$
}

\begin{abstract}
RESUMO
Objetivo: Identificar ações de Enfermagem que contribuem para o preparo da colonoscopia, de modo a subsidiar boas práticas de enfermagem nos serviços de endoscopia e minimizar riscos relacionados ao preparo inadequado. Métodos: Revisão integrativa com busca nas bases PUBMED, Web of Science, SCOPUS e CINAHL, realizada no período de julho e agosto de 2020.Após aplicação dos critérios estabelecidos, foram elegíveis cinco estudos para a revisão. Resultados:As principais ações de enfermagem descritas foram educativas, sendo utilizadas metodologias ativas, personalizada e direcionada ao usuário, palestras e/ou metodologias passivas.Ações gerenciais estiveram presentes, sendo valorizadas a comunicação entre a equipe e o desenvolvimento de um aplicativo de orientações educativas. Por fim, foram descritas ações assistenciais relativas ao aprazamento e administração de medicações do preparo para a colonoscopia. Conclusão:A educação em saúde destacou-se como principal ação de enfermagem direcionada ao preparo do exame de colonoscopia. No que tange às estratégias utilizadas, metodologias lúdicas e problematizadoras possuem maior eficácia quando comparadas às tradicionais. $\bigcirc$ enfermeiro possui papel fundamental no campo da educação em saúde, e, tal protagonismo deve ser não apenas mantido, mas também estimulado, com a finalidade de realçar sua missão dentro da equipe de saúde.
\end{abstract}

Palavras-chave: Cuidados de Enfermagem; Colonoscopia; Segurança do Paciente.

\begin{abstract}
Objective: Identify nursing actions that contribute to the preparation of colonoscopy, in order to support good nursing practices in endoscopy services and minimize risks related to inadequate preparation. Methods: Integrative review with search in the PUBMED, Web of Science, SCOPUS and CINAHL databases, carried out between July and August 2020. After applying the established criteria, five studies were eligible for the review. Results: The main nursing actions described were educational, using active, personalized and user-oriented methodologies, lectures and / or passive methodologies. Management actions were present, with communication between the team and the development of an educational guidance application being valued. Finally, assistance actions related to the scheduling and administration of medications in preparation for colonoscopy were described. Conclusion: Health education stood out as the main nursing action aimed at preparing the colonoscopy exam. Regarding the strategies used, playful and problematizing methodologies are more effective when compared to traditional ones. The nurse has a fundamental role in the field of health education, and such a role must not only be maintained, but also encouraged, in order to enhance their mission within the health team.
\end{abstract}

Key-words: Nursing Care; Colonoscopy; Patient Safety.

I Doutora em Enfermagem. Docente da Faculdade de Enfermagem e do Programa de Pós-Graduação - Mestrado em Enfermagem da Universidade Federal de Juiz de Fora. Líder do Grupo de Pesquisa Enfermagem e o cuidado à saúde de pessoas, famílias e coletividades em todo o ciclo vital. Coordenadora do Projeto de Extensão Educação em Saúde em face do câncer colorretal e do exame de colonoscopia: contribuições da Enfermagem.

2 Acadêmico de Enfermagem. Faculdade de Enfermagem da Universidade Federal de Juiz de Fora. Bolsista do Projeto de Extensão Educação em Saúde em face do câncer colorretal e do exame de colonoscopia: contribuições da Enfermagem.

3 Acadêmico de Enfermagem. Faculdade de Enfermagem da Universidade Federal de Juiz de Fora.

4 Doutora em Enfermagem. Docente da Faculdade de Enfermagem. Líder do Grupo de Pesquisa Enfermagem e o cuidado à saúde de pessoas, famílias e coletividades em todo o ciclo vital.Vice-coordenadora do Projeto de Extensão Educação em Saúde em face do câncer colorretal e do exame de colonoscopia: contribuições da Enfermagem.

5 Doutora em Enfermagem. Docente do Programa de Pós-Graduação - Mestrado em Enfermagem da Universidade Federal de Juiz de Fora. Membro do Grupo de Pesquisa Enfermagem e o cuidado à saúde de pessoas, famílias e coletividades em todo o ciclo vital.

6 Mestranda em Enfermagem. Programa de Pós-Graduação - Mestrado em Enfermagem da Universidade Federal de Juiz de Fora.

7 Mestranda em Enfermagem. Programa de Pós-Graduação - Mestrado em Enfermagem da Universidade Federal de Juiz de Fora.

8 Mestranda em Enfermagem. Programa de Pós-Graduação - Mestrado em Enfermagem da Universidade Federal de Juiz de Fora.

9 Mestranda em Enfermagem. Programa de Pós-Graduação - Mestrado em Enfermagem da Universidade Federal de Juiz de Fora.

10 Acadêmico de Enfermagem. Faculdade de Enfermagem da Universidade Federal de Juiz de Fora. 


\section{INTRODUÇÃO}

A incidência e mortalidade por neoplasias de cólon e reto tendem a aumentar nas próximas décadas. Em 2018, quase dois milhões de diagnósticos e um milhão de mortes foram esperadas no mundo. A exacerbação da doença se deve especialmente a fatores relacionados a dieta e sedentarismo. Em países em desenvolvimento, como o Brasil, aspectos socioeconômicos revelam-se determinantes para o aumento de casos novos ${ }^{(1)}$, os quais em 2020 ocupam o segundo lugar das neoplasias mais incidentes ${ }^{(2-3)}$.

Embora haja diminuição dessa taxa na população idosa, houve aumento em adultos com idades de 50 anos ou menos, sendo diagnosticados para cada dez pessoas, uma nessa faixa etária. Chama a atenção o fato de três em cada quatro adultos com diagnóstico de câncer colorretal não terem história familiar da doença, o que aponta para o benefício da detecção precoce por meio do rastreamento ${ }^{(3)}$.

Nesse contexto, recomenda-se a realização da colonoscopia a partir dos 50 anos para pessoas sem histórico familiar de alto risco, exceção feita aos afroamericanos, nas quais evidências apoiam a triagem a partir dos 45 anos. A principal vantagem da colonoscopia em relação a outros métodos de rastreamento é a de que o diagnóstico e o tratamento - polipectomia de lesões précancerosas - podem ser realizados na mesma sessão. No entanto, as desvantagens incluem riscos relativos ao procedimento, tais como sangramento, perfuração e aspiração; expertise do endoscopista na detecção de adenoma, e a necessidade de preparação intestinal ${ }^{(3-5)}$.

A preparação intestinal, que geralmente consiste em mudanças na dieta desde a véspera, ingestão aumentada de líquidos e uso de medicamentos laxantes, contribui significativamente para a qualidade do exame de colonoscopia quando realizada de modo adequado. Do contrário eleva o custo por requerer repetição do procedimento, impacta negativamente para o sucesso da intubação cecal, prolonga o tempo de procedimento, proporciona maior índice de complicações, limita a sensibilidade para identificação de pólipos e lesões, as quais sob análise histopatológica poderiam confirmar a presença de neoplasias, com início precoce do tratamento ${ }^{(6-9)}$. Especialmente em idosos deve haver maior vigilância, uma vez que o preparo intestinal inadequado relaciona-se sobremaneira à não conformidade com orientações dietéticas e de ingestão da medicação laxativa ${ }^{(10)}$.

Nesse contexto, evidências apontam que intervenções educativas provenientes da equipe multiprofissional, melhoram a qualidade de limpeza do cólon e propiciam resultados colonoscópicos relevantes, reduzindo a taxa de inadequação do preparo ${ }^{(8,11)}$. Nos serviços de endoscopia, o enfermeiro possui competência para realizar funções de cunho técnico-assistencial e de gerenciamento do cuidado no que tange ao preparo intestinal, aumentando a adesão do usuário e favorecendo a qualidade do exame de colonoscopia ${ }^{(12)}$.

Assim, propõe-se a realização de uma revisão integrativa com o objetivo de identificar ações de Enfermagem que contribuem para o preparo da colonoscopia, de modo a subsidiar boas práticas de enfermagem nos serviços de endoscopia, minimizar riscos relacionados ao preparo inadequado, otimizando a segurança da assistência prestada.

\section{METODOLOGIA}

Estudo de revisão integrativa da literatura, com análise ampla de estudos publicados que possibilitam discussões sobre métodos e resultados de pesquisa. Foram consideradas seis etapas, a saber: definição da questão norteadora; busca dos estudos na literatura; coleta de dados; avaliação do conteúdo selecionado; discussão; e publicação dos resultados ${ }^{(13-14)}$.

Utilizou-se da estratégia $\mathrm{PICO}^{(15)}$ para adequar à formulação da questão de pesquisa, sendo essa delimitada como: quais ações de Enfermagem contribuem para o preparo da colonoscopia, de modo a subsidiar boas práticas de enfermagem nos serviços de endoscopia, minimizar riscos relacionados ao preparo inadequado, otimizando a segurança da assistência prestada?

As buscas nas bases de dados National Institutes of Health (PUBMED), Web of Science, SCOPUS e CINAHL foi realizada por dois revisores de modo independente e concomitante no período de julho e agosto de 2020 , utilizando os descritores com a estratégia de busca (tw:(colonoscopy) AND bowel preparation AND (nursing care OR nursing) (tw:(colonoscopy)) AND (tw:(bowel preparation)) AND (tw:(nursing care OR nursing)), conforme terminologia dos Descritores em Ciências da Saúde (DeCS) e Medical Subject Headings (MeSH).

Os critérios de inclusão consideraram artigos que evidenciaram ações de enfermagem voltadas ao preparo da colonoscopia; publicados em periódicos nacionais ou internacionais nos últimos dez anos (2010-2020); disponíveis eletronicamente na íntegra, nos idiomas inglês, português e espanhol. Excluíram-se capítulos de livros, editoriais, resumos, cartas ao editor, artigos de reflexão, revisão, atualização, dissertações e teses.

A seleção dos estudos ocorreu por meio da leitura de títulos e resumos, sendo selecionados os que atendiam aos critérios de inclusão. Esse processo foi realizado por dois revisores e somente quando havia divergência, um terceiro revisor era consultado.

Foram encontrados 220 estudos por meio da busca nas bases de dados, sendo identificados 39 após a aplicação dos critérios e remoção das duplicatas. A partir 
da leitura dos títulos e resumos, a amostra reduziu para sete artigos cuja leitura na íntegra apontou a exclusão de dois, selecionando-se finalmente cinco estudos para composição dos resultados da revisão.

A Figura I apresenta o fluxograma de seleção dos estudos. Um formulário previamente elaborado pelas autoras foi utilizado para extração dos dados dos artigos, contendo autores, ano e país de publicação, objetivo do estudo, delineamento metodológico, nível de evidência ${ }^{(14)}$ e principais ações de enfermagem.

\section{RESULTADOS E DISCUSSÃO}

Os cinco artigos incluídos foram alocados em uma matriz de análise com dados e informações condizentes ao interesse dessa revisão. No Quadro 0I, está disposta a caracterização dos artigos cujas ações de enfermagem contribuíram para o preparo intestinal de pacientes submetidos à colonoscopia.

Dois estudos foram produzidos na China (E5 e E3), um nos Estados Unidos (EI), outro na Holanda (E2) e por fim, em Portugal (E4). Relativo ao ano de publicação, um estudo foi publicado em 2014 (EI), um em 2018 (E3), dois em 2017 (E2 e E4) e um em 2020 (E5).

O delineamento metodológico que prevaleceu foi de estudo experimental (E3, E4 e E5) seguido de estudo quase experimental (E2) e por fim, estudo observacional (EI).

A classificação do nível de evidência a maioria foi classificada em nível 2 (E3, E4, e E5), seguido do nível 3 (EI e E2).

As principais ações de enfermagem descritas nos estudos foram educativas (E2, E3, E4 e E5), sendo utilizadas metodologias ativas (E3), personalizada e direcionada ao

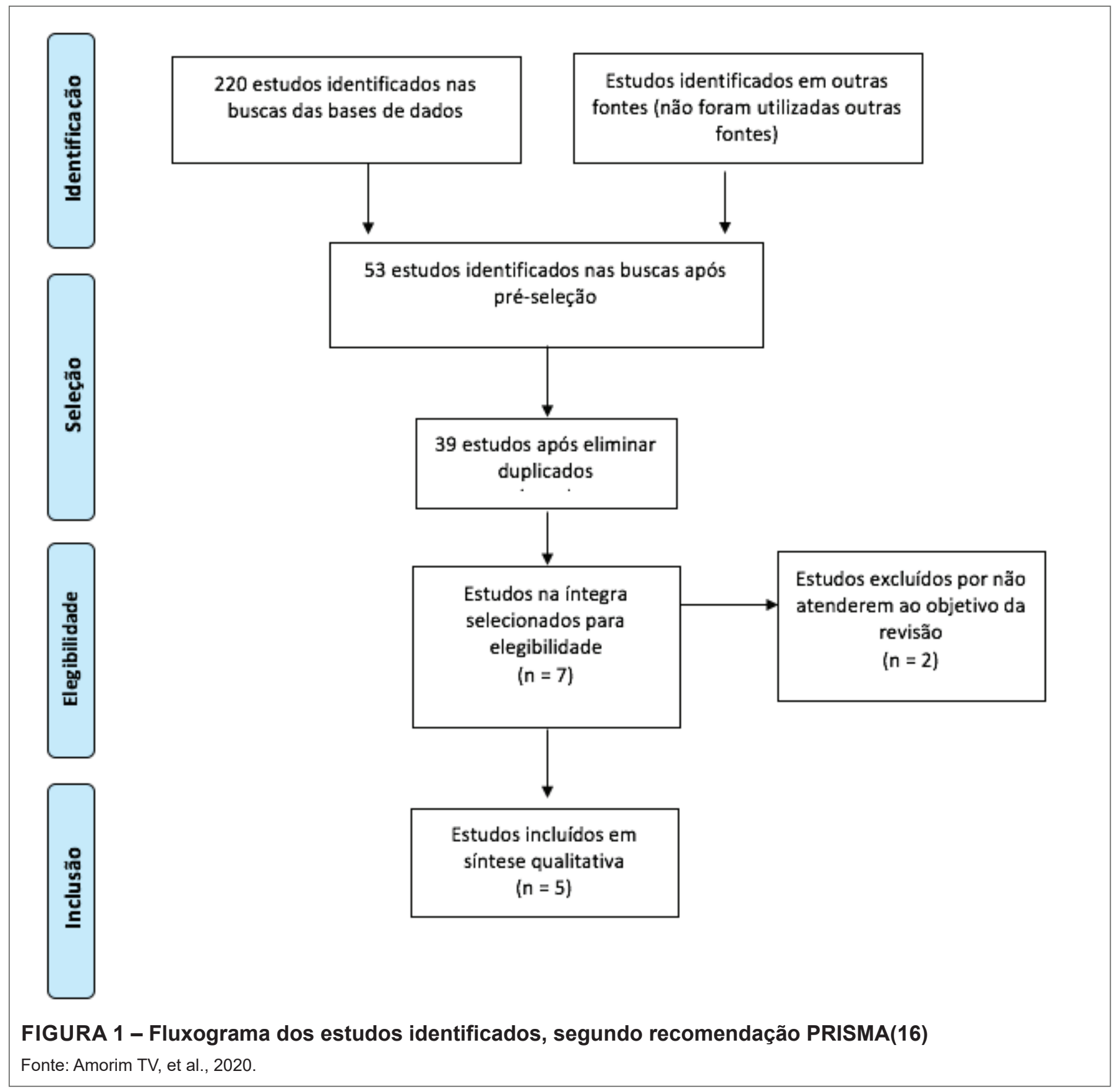




\section{QUADRO 01 - Compilação e síntese dos estudos selecionados}

\begin{tabular}{|c|c|c|c|c|}
\hline Estudo & $\begin{array}{l}\text { Autores } \\
\text { Ano }\end{array}$ & País & Objetivos & $\begin{array}{l}\text { Método e } \\
\text { Nível de evidência } \\
\text { (NE) }\end{array}$ \\
\hline$E 1^{17}$ & $\begin{array}{l}\text { Yang D, } \\
\text { Summerlee R, Rajca B, } \\
\text { et al. } \\
2014\end{array}$ & EUA & $\begin{array}{l}\text { Compreender as barreiras percebidas à } \\
\text { administração de doses divididas entre a } \\
\text { enfermagem e os prestadores. }\end{array}$ & $\begin{array}{l}\text { Estudo observacional } \\
\text { NE: } \mathbf{3}\end{array}$ \\
\hline$E 2^{18}$ & $\begin{array}{l}\text { Veldhuijzen G, Klemt- } \\
\text { Kropp M, Noomen C. } \\
\qquad \begin{array}{l}\text { et al. } \\
2017\end{array}\end{array}$ & Holanda & $\begin{array}{l}\text { Verificar a eficácia da instrução assistida por } \\
\text { computador (CAI) na educação do paciente antes } \\
\text { da colonoscopia, medindo a limpeza intestinal e o } \\
\text { conforto e a ansiedade do paciente. }\end{array}$ & $\begin{array}{l}\text { Estudo quase- } \\
\text { experimental } \\
\text { NE:3 }\end{array}$ \\
\hline$E 3^{19}$ & $\begin{array}{c}\text { Liu C, Song X, Hao H, } \\
2018\end{array}$ & China & $\begin{array}{l}\text { Avaliar a qualidade da preparação intestinal, } \\
\text { através da escala de preparação intestinal de } \\
\text { Ottawa; e avaliar o efeito de assistir a um vídeo } \\
\text { simples seguido de pacientes recontando o } \\
\text { processo de preparação do intestino. }\end{array}$ & $\begin{array}{l}\text { Estudo experimental } \\
\qquad \mathrm{NE}: 2\end{array}$ \\
\hline $\mathrm{E} 4^{20}$ & $\begin{array}{l}\text { Elvas L, Brito D, Areia } \\
\qquad \begin{array}{c}\text { M, et al. } \\
2017\end{array}\end{array}$ & Portugal & $\begin{array}{l}\text { Analisar o impacto da educação personalizada do } \\
\text { paciente na preparação da limpeza intestinal para } \\
\text { colonoscopia. }\end{array}$ & $\begin{array}{l}\text { Estudo experimental } \\
\qquad \mathbf{N E : 2}\end{array}$ \\
\hline$E 5^{21}$ & $\begin{array}{c}\text { Guo B, Zuo X Li Z. et al. } \\
\qquad 2020\end{array}$ & China & $\begin{array}{l}\text { Explorar se a informação educacional fornecida } \\
\text { através de um smartphone médico em conjunto } \\
\text { com instruções verbais e escritas, em comparação } \\
\text { com as tradicionais instruções verbais e baseadas } \\
\text { em livros, poderia melhorar a qualidade da } \\
\text { preparação intestinal de pacientes hospitalizados } \\
\text { submetidos a colonoscopia. }\end{array}$ & $\begin{array}{l}\text { Estudo experimental } \\
\qquad \mathrm{NE:2}\end{array}$ \\
\hline
\end{tabular}

Fonte: Amorim TV, et al., 2020.

\section{QUADRO 02 - Principais resultados dos estudos selecionados}

\begin{tabular}{|l|l|}
\hline Estudo & Principais ações de Enfermagem \\
\hline E117 & $\begin{array}{l}\text { As principais ações foram de cunho assistencial, haja visto a administração dividida das doses de polietilenoglicol e, de caráter } \\
\text { gerencial, no que tange à comunicação direta entre a equipe de enfermagem, garantindo a continuidade da assistência. }\end{array}$ \\
\hline E218 & $\begin{array}{l}\text { Ações educativas, incluindo o aconselhamento de enfermagem, além da instrução assistida por computador, sendo um importante } \\
\text { instrumento educativo. }\end{array}$ \\
\hline E319 & Ações educativas, com uso de metodologia ativa visando melhor fixação do conteúdo. \\
\hline E420 & Ações educativas, de forma personalizada e direcionada aos pacientes. \\
\hline E521 & $\begin{array}{l}\text { Ações educativas e gerenciais, respectivamente por meio de orientações verbais aos pacientes e por meio do desenvolvimento } \\
\text { de um aplicativo. }\end{array}$ \\
\hline
\end{tabular}

Fonte: Amorim TV, et al., 2020.

usuário (E4), palestras e/ou metodologias passivas (E2 e E5).Ações gerenciais estiveram presentes (EI e E5), sendo essas, comunicação entre a equipe (EI), e o desenvolvimento de um aplicativo de orientações educativas(E5). Por fim, ações assistenciais (EI) relativas ao aprazamento e administração de medicações do preparo para a colonoscopia. O Quadro 02 destaca a síntese das principais ações de enfermagem.

Os enfermeiros sendo profissionais direcionados à educação em saúde, vem fazendo uso de tecnologias para mediar o cuidado prestado(22). De modo geral, os artigos destacaram a importância da educação em saúde para o preparo adequado(|8-21), com a necessária adaptação à linguagem do paciente, esclarecendo dúvidas pertinentes. $\mathrm{O}$ uso de ferramentas de metodologia ativa auxiliou para maior fixação do protocolo de preparo. Destacouse nesse sentido, folheto ou livreto com instruções ao paciente ${ }^{(19-21)}$, configurando-se como estratégias importantes de consulta dos pacientes em seus domicílios.

A educação em saúde por meio de tecnologias educa- 
tivas - folhetos, cartilhas, dentre outros - advém tal qual instrumento de apoio às equipes de saúde, em especial o enfermeiro. $O$ cuidado de enfermagem deve ser pautado na educação em saúde por meio de orientação individual em consonância às suas necessidades, sendo o estreitamento de vínculos imprescindível para a troca de saberes ${ }^{(23-24)}$. Essas ações se mostraram favoráveis a um melhor preparo e maiores taxas de sucesso no resultado do exame ${ }^{(21)}$, em especial, quando a intervenção educativa é individualizada ${ }^{(20)}$.

Algumas metodologias são mais utilizadas pela enfermagem na abordagem educação em saúde, entretanto, possuem um caráter tradicionalista focado exclusivamente na tentativa de mudança comportamental ${ }^{(25)}$.Apenas um estudo utilizou de tal estratégia ${ }^{(21)}$, embora não o tenha feito de forma exclusiva, e sim como apoio ao aplicativo desenvolvido. Tal questão aponta para o fato de que metodologias exclusivamente passivas, utilizadas de forma unidirecional e por meio da transmissão de informações, não se mostram tão eficazes quando comparadas às metodologias ativas.

Nesse contexto, considera-se que palestras podem limitar o aprendizado, desestimulando a interação entre os ouvintes, por não haver problematização e diálogo entre os participantes. Já as rodas de conversa com o objetivo de esclarecer dúvidas sobre a doença e/ou tratamento, bem como favorecer a integração entre usuários e equipe, associados ao uso de materiais didáticos, mostraram-se importantes para o sucesso das práticas educativas ${ }^{(26)}$.

Em nível hospitalar, embora seja consenso entre a equipe de saúde, a importância do preparo intestinal para a colonoscopia, esse ainda se evidencia como um desafio a ser superado pela díade profissional-paciente ${ }^{(17)}$. Para uma assistência de enfermagem de qualidade no âmbito da colonoscopia, a equipe de enfermagem deve ter conhecimento e compreensão acerca do preparo intestinal nos aspectos da farmacocinética das medicações utilizadas, bem como quais efeitos têm sobre os pacientes ${ }^{(27)}$.

Tal fato traz à luz a necessidade de maior investimento na educação em saúde no ambiente hospitalar, além da fluência na comunicação entre os membros da equipe de enfermagem, posto que as intervenções educacionais melhoram significativamente a qualidade do preparo do colón de pacientes internados ${ }^{(28)}$.

A educação e treinamento da equipe de enfermagem e, em especial, a de enfermagem, deve ser enfatizada e realizada constantemente ${ }^{(17)}$. Evidências de um ensaio clínico apontaram a importância do treinamento para enfermeiros assistenciais lotados em hospitais para melhorar a qualidade do preparo intestinal e aspectos que envolvem a subjetividade de pacientes diante da colonoscopia ${ }^{(29)}$.

Para além da educação em saúde direcionada ao exame, deve-se ainda atentar para a vistas à conscientização da população em relação à prevenção do câncer colorretal pela prevalência e incidência aumentadas. Nesse sentido, intervenção educativa seriada mostrou-se eficaz no reconhecimento da população acerca dos benefícios do exame de rastreio, bem como um nível de conhecimento significativamente maior relacionado à sensibilidade e percepções de gravidade, triagem por meio de teste de sangue oculto nas fezes e intenção de se submeter a triagem por colonoscopia ou sigmoidoscopia ${ }^{(30)}$.

\section{CONCLUSÃO}

A educação em saúde destacou-se como principal ação de enfermagem direcionada ao preparo da colonoscopia, devendo ser estimulada entre a equipe de enfermagem a fim de melhorar o resultado do exame. No que tange às estratégias utilizadas, metodologias lúdicas e problematizadoras possuem eficácia maior quando comparadas às tradicionais. $O$ enfermeiro possui um papel fundamental no campo da educação em saúde, e, tal protagonismo deve ser não apenas mantido, mas também estimulado, com a finalidade de realçar sua missão dentro da equipe de saúde. Durante a assistência e prestação de cuidados no contexto da colonoscopia, algumas metodologias de ações educativas podem ser utilizadas pela equipe de enfermagem a fim de utilizar o momento para promover a saúde além de enfatizar a importância do preparo do exame. $O$ presente estudo de revisão apresentou como limitação o número reduzido de publicações relacionadas à temática pretendida, constituindo-se assim lacuna do conhecimento produzido por enfermeiros, especialmente no âmbito nacional. 


\section{REFERÊNCIAS}

I. Rawla P, Sunkara T, Barsouk A. Epidemiology of colorectal cancer: incidence, mortality, survival, and risk factors. Prz Gastroenterol. 2019 [acesso em 12 jul 2020]; I4(2): 89- 103. Disponível em: 10.5 I |4/pg.2018.81072.

2. Siegel RL, Miller KD, Goding Sauer A, Fedewa SA, Butterly LF, Anderson JC, et al. Colorectal cancer statistics, 2020. CA Cancer J Clin. 2020 [acesso em I4 jul 2020]; 70(3): I45I64. Disponível em: https://doi.org/ 10.3322/caac.21601.

3. Instituto Nacional De Câncer José Alencar Gomes Da Silva. 2019. Estimativa 2020: Incidência de câncer no Brasil / Instituto Nacional de Câncer José Alencar Gomes da Silva. Rio de Janeiro. Disponível em: https:/www.inca.gov.br/sites/ ufu.sti.inca.local/files/media/document/estimativa-2020-incidencia-de-cancer-no-brasil.pdf.Acesso em I3 out. 2020.

4. Leal RM, Mendes CRS, Moreira LF, Amorim TMB, Andrade ACM, Gonçalves ES, Mendes CRS. Achados colonoscópicos em pacientes a partir dos 50 anos: uma análise crítica de I.6I4 exames. Journal of Coloproctoogy. 2019 [acesso em 8 ago 2020]; 39(I): 22-26. Disponível em: https://doi. org/l0.1016/j.jcol.2018.09.006.

5. Gupta N, Kupfer SS, Davis AM. Colorectal Cancer Screening. JAMA. 2019 [acesso em 2 ago 2020]; 32I (20): 2022-2023. Disponível em: https://doi.org/10.1001/jama.2019.4842.

6. Coser RB, Dalio MB, Martins LCP, Alvarenga GF, Cruz CA, Imperiale AR, Padovese CC, Paulo GA, Júnior JCT. Colonoscopy complications: experience with 8968 consecutive patients in a single institution. Rev Col Bras Cir. 2018 [acesso em 06 set 2020];45(4):el 896. Disponível em: https://doi. org./I0.1590/0100-6991 e-20181858.

7. Nisihara R, Fudalli F, Turra MLM, Malanche RM, Benassi SH, Pimenta APP, Sakamoto DG. Pathological findings of colorectal polyps analyzed in Curitiba - Brazil. Journal of Coloproctology. 2017 [acesso em 15 ago 2020]; 37(2): I23-I27. Disponível em: https://doi.org/l0.1016/j.jcol.2017.02.00I.

8. Gkolfakis P, Tziatzios G, Papanikolaou IS, Triantafyllou K. Strategies to improve inpatients quality of bowel preparation for colonoscopy: a systematic review and meta-analysis. Gastroenterology Research and Practice. 2019 [acesso em II ago 2020]; (2019): I-I5. Disponível em: https://doi. org/10.1155/2019/5 |47208.

9. Fayad NF, Kahid CJ. Colonoscopy quality assessment. Gastrointest Endosc Clin N Am. 2015 [acesso 20 set 2020]; 25(2): 373-86. Disponível em: https://doi.org/10.1016/j. giec.20I4.II.008.

10. Zhang YY, Niu M,Wu ZY,Wang XY, Zhao YY, Gu J.The incidence of and risk factors for inadequate bowel preparation in elderly patients:A prospective observational study. Saudi J Gastroenterol. 2018 [acesso em II set 2020]; 24(2):8792. Disponível em: https://doi.org/I0.4I03/sjg.SJG_426_I7.

I I. Lee YJ, Kim ES, Park KS, Cho KB, Jang BK, Chung WJ, et al. S Education for Ward Nurses Influences the Quality of Inpatient's Bowel Preparation for Colonoscopy. Medicine. 2015 [acesso em I 3 jul 2020]; (94): e l 423. Disponível em: https:// doi.org//0.1097/MD.0000000000001 423.

12. Diniz TSR. Construção de folheto educativo e orientação via telefone para o preparo da colonoscopia: estudo clínico, controlado e randomizado [dissertação]. São Paulo: Universidade Estadual Paulista; 2019.

13. Whittemore R, Knafl K. The integrative review: up dated methodology. J Adv Nurs. 2005 [acesso em 20 set 2020]; 52(5): 546-53. Disponível em: http://dx.doi.org/I0.1III/j. |365-2648.2005.0362|.x.

14. Mendes KDS, Silveira RCDCP, Galvão CM. Revisão integrativa: método de pesquisa para a incorporação de evidências na saúde e na enfermagem. Texto Contexto Enferm. 2008 [acesso em 8 jul 2020]; 17(4): 758-64. Disponível em: http://dx.doi.org/ I 0.I 590/S0 I 04-070720080004000 I8.

I5. Ercole FF, Melo LS, Alcoforado CLGC. Revisão Integrativa versus Revisão Sistemática. Revista Mineira de Enfermagem. 2014 [acesso em 22 set 2020]; I8(I): I-I I. Disponível em: https://doi.org//0.5935/I4I5-2762.20 I4000 I.

16. Moher D, Liberati A, Tetzlaff J, Altman DG. Principais itens para relatar Revisões sistemáticas e Meta-análises: A recomendação PRISMA*. Epidemiol. Serv. Saúde. 2015 [acesso em 20 set 2020]; 24(2): 335-342. Disponível em: https://doi. org/I0.5I23/5I679-497420I50002000I7.

17. Yang D, Summerlee R, Rajca B, Williamson JB, LeLaurin J, McClellan L, et al.A pilot study to evaluate the feasibility of implementing a split-dose bowel preparation for inpatient colonoscopy:a single-center experience. BMJ Open Gastroenterol. 20 I 5 [acesso em 5 jul 2020]; I ( I): e000006. Disponível em: http://dx.doi.org/I0. I |36/bmjgast-20|4-000006.

18. Veldhuijzen G, Klemt-Kropp M, Noomen C, Van Esch AA, Tjwa ET, Drenth J. Computer-assisted instruction before colonoscopy is as effective as nurse counselling, a clinical pilot trial. Endoscopy international open. 2017 [acesso em 21 jul 2020]; 5(8): E792. Disponível em: http://dx.doi.org/l0.1055/s-0043-I 108I3.

19. Liu C, Song X, Hao H. Educational Video Followed by Retelling Bowel Preparation Process to Improve Colonoscopy Bowel Preparation Quality: A Prospective Nursing Intervention Study. Medical science monitor. 2018 [acesso em I4 set 2020]; (24): 6029. Disponível em: http://dx.doi. org/10.12659/MSM.909572.

20. Elvas L, Brito D, Areia M, Carvalho R, Alves S, Saraiva S, Cadime AT. Impact of personalised patient education on bowel preparation for colonoscopy: prospective randomised controlled trial. GE-Portuguese Journal of Gastroenterology. 2017 [acesso em 24 jul 2020]; 24(I): 22-30. Disponível em: http://dx.doi.org/10.I 159/000450594.

21. Guo B, Zuo X, Li Z, Liu J, Xu N, Li X, Zhu A. Improving the quality of bowel preparation through an app for inpatients undergoing colonoscopy: A randomized controlled trial. J Adv Nurs. 2020 [acesso em 28 set 2020]; 76: 1037-1045. Disponível em: https://doi.org/ I0.1 I I I/jan. 14295. 
22. Lima ACMACC, Bezerra KDC, Sousa DMDN, Vasconcelos CTM, Coutinho JFV, Oriá MOB. Educational technologies and practices for prevention of vertical HIV transmission. Revista Brasileira de Enfermagem. 2018 [acesso em II jul 2020]; 7I: I759-67. Disponível em: https://doi.org//0.1590/ S0I02-3 I IX200700I500005.

23. Saldan GG, Figueiredo FSF, Misawa F, Rêgo AS, Salci MA, Radovanovic CAT. Construction of educational technology for home care after encephalic vascular accident: case report. Rev Enferm UFPE. 2017 [acesso em 3 ago 2020]; I I (4): 1784-93. Disponível em: https://doi.org//0.5205//98|8963-v I li4a I525 I I 784-I793-20 I7.

24. Farias DLSD, Nery RNB, Santana MED. O enfermeiro como educador em saúde da pessoa estomizada com câncer colorretal. Enferm. Foco. 2019 [acesso em 7 ago 2020]; 10 (I) 35-39. Disponível em: revista.cofen.gov.br/index.php/enfermagem/article/view//486/490.

25. Dodou HD, Oliveira TDAD, Oriá MOB, Rodrigues DP, Pinheiro PNDC, Luna IT. La práctica educativa realizada por la enfermería en el puerperio: representaciones sociales de puérperas. Revista Brasileira de Enfermagem. 2017 [acesso em 27 set 2020]; 70(6): I250- I 258. Disponível em: https:// doi.org/I0.1590/0034-7I67-2016-0136.

26. Azevedo PRD, Sousa MMD, Souza NFD, Oliveira SHDS. Ações de educação em saúde no contexto das doenças crônicas: revisão integrativa. Revista online de pesquisa.
2018 [acesso em 21 de set 2020]; 260-267. Disponível em: https://doi.org/l0.9789/2I75-536I.20I8.vI0il.260-267.

27. Mangnall R. Good bowel cleaning vital for effective colonoscopy. Kai Tiaki: Nursing New Zealand. 2012 [acesso em 25 jul 2020]; 18(6): I7- I 9. Disponível em: researchgate.net/ publication/230738285_Good_bowel_cleaning_vital_for_ effective_colonoscopy.

28. Gkolfakis P,Tziatzios G, Papanikolau IS, Triantafyllou K. Strategies to Improve Inpatients' Quality of Bowel Preparation for Colonoscopy: A Systematic Review and Meta-Analysis. Gastroenterology Research and Pratice. 2019 [acesso em 10 ago 2020];2019: I-I5. Disponível em: https://doi. org/I0.I I55/2019/5।47208.

29. Lee YJ, Kim ES, Park KS, Cho KB, Jang BK, Chung WJ, Hwang JS. Education for Ward Nurses Influences the Quality of Inpatient's Bowel Preparation for Colonoscopy. Medicine. 2015 [acesso em 28 jul 2020]; 94(34): I-9. Disponível em: https://doi.org/10.1097/ MD.000000000000| 423.

30. Abuadas FH, Nustas WJP, Abuadas MH. The Effect of a Health Education Intervention on Jordanian Participants' Colorectal Cancer Knowledge, Health Perceptions, and Screening Practices. Cancer Nurs. 20I 8; 4 I (3):226-237.

Recebido: 2020-|0-2|

Aceito: 2020-II-04 